\title{
Growth Dynamics of Crystalline Tensionless Surfaces
}

\author{
Esteban Moro,* Rodolfo Cuerno, ${ }^{\dagger}$ and Angel Sánchez ${ }^{*}$ \\ Grupo Interdisciplinar de Sistemas Complicados, Departamento de Matemáticas, Universidad Carlos III de Madrid, \\ c/ Butarque 15, E-28911 Leganés, Madrid, Spain
}

(Received 2 December 1996)

\begin{abstract}
We introduce a model for two-dimensional crystalline tensionless surfaces. In equilibrium, the model exhibits a roughening transition to the high temperature phase of the sine-Gordon (sG) model, though its initial stages are rougher and described by the linear molecular beam epitaxy equation; the transition temperature is about half the sG one. Depending on distance to equilibrium and temperature, the model can behave as a nonmoving flat interface, a moving interface with oscillatory roughness, or a rough moving interface. Possible experimental applications are discussed. [S0031-9007(97)03495-9]
\end{abstract}

PACS numbers: 68.35.Rh, 81.10.Aj, 81.10.Bk, 81.15.Hi

Surface and interface roughness are crucial in determining the properties and technological applicability of many systems [1]. Rough surfaces develop as a consequence of competition among different effects, such as surface tension, surface diffusion, thermal fluctuations, lattice effects, applied forces, and so on. Thus, in equilibrium a particular surface can be rough under certain conditions and macroscopically "flat" under other ones, these two regimes being usually separated by a roughening transition [1,2]. Out of equilibrium, surfaces can grow in many different modes, ranging from layer by layer (LBL) to rough growth: Thus, ordered LBL growth is detected experimentally in a number of growth processes as, for instance, oscillations in the reflection high-energy electron diffraction (RHEED) specular intensity [3], whereas for a growing rough interface the coherence leading to RHEED oscillations is lost.

Among recent studies of surface dynamics, systems with negligible surface tension have received much attention. In equilibrium, particularly interesting examples are flexible membranes, for which the energy of deformations around the flat configuration is a function of the surface curvature (the so called Helfrich functional) [2]. Out of equilibrium, tensionless surfaces are expected, for instance, in thin films grown by molecular beam epitaxy (MBE) [1,3,4]: Villain [5] and Lai and Das Sarma [6], elaborating on classic works by Herring and by Mullins [7], proposed that surfaces grown by MBE obey relaxation mechanisms that locally minimize surface curvature; to linear order, this coincides with the behavior of the tensionless membranes referred to above and is encompassed in the so called linear MBE equation for surface height [5,6]. Recent experiments described by this equation, such as [8,9], are discussed below.

In this Letter, we study both equilibrium and out-ofequilibrium tensionless surfaces in a unified framework, focusing on their behavior in the presence of lattice or discreteness effects. To this end, we propose a model given by the Hamiltonian

$$
\begin{aligned}
\mathcal{H}= & \frac{\kappa}{2} \sum_{i}\left[\left(\sum_{\mathrm{nn}} h_{j}\right)-n h_{i}\right]^{2} \\
& -V_{0} \sum_{i} \cos \left(h_{i} / a\right)-\sum_{i} I_{i} h_{i},
\end{aligned}
$$

where the (single valued, continuously varying) functions $h_{i}(t)$ give the height above site $i$ in a two-dimensional (2D) square $(L \times L)$ lattice, the sum in the brackets runs over the nearest neighbors of site $i, n=4$ being the lattice coordination number, and $a$ is the vertical lattice spacing.

Let us discuss the physical meaning of the terms in (1). The first one is a discrete version of the Laplacian squared of the height, which favors small surface curvatures and corresponds, e.g., to the lowest order term in the Helfrich free energy for a tensionless membrane [2]. The second term, a weighting function favoring $h_{i}$ to be $2 n \pi a$, is a pinning potential that represents lattice effects or, equivalently, the discreteness of growth events taking place. This is a nontrivial result first found by Chui and Weeks in the study of a continuous version of the discrete Gaussian model [10]; see also [11,12]. Specifically, it has been shown in [10] that the partition function of the discrete Gaussian model [13] (in which the height takes only discrete values $\left.h_{i}=0, \pm a, \pm 2 a, \ldots\right)$ is proportional to that of the same model with an additional cosine term and no constraint on the values of $h_{i}$ (i.e., $h_{i}$ become continuously varying real functions). In this way the discrete Gaussian model is shown to have the same universal properties as the sine-Gordon (sG) [14] model. The same approach yields the second term in Eq. (1); this mapping has been also successfully applied in other contexts to study the interplay of discrete growth events with different relaxation mechanisms [15]. Finally, the third term in (1) explicitly includes nonequilibrium effects through the interaction of the surface with applied fields $I_{i}$ which represent, e.g., the chemical potential due to the flux of incoming particles $[1,11,12]$. 
To study model (1) we resort to Langevin dynamics, a technique successfully applied to the equilibrium and nonequilibrium properties of the sG model [16]. We integrate the overdamped equations of motion for $h_{i}$ (rescaled as to have $\kappa=a=V_{0}=1$ ):

$$
\frac{\partial h_{i}}{\partial t}=-\nabla_{d}^{2}\left(\nabla_{d}^{2} h_{i}\right)-\sin h_{i}+I_{i}+\xi_{i}(t)
$$

$\nabla_{d}^{2}$ stands for the discrete Laplacian in the square lattice and $\xi_{i}(t)$ are independent Gaussian white noises of zero mean and $\left\langle\xi_{i}(t) \xi_{j}\left(t^{\prime}\right)\right\rangle=2 T \delta_{i j} \delta\left(t-t^{\prime}\right), T$ being the temperature. Equation (2) reproduces the long distance behavior of the continuum equation

$$
\frac{\partial h}{\partial t}=-\nabla^{2}\left(\nabla^{2} h\right)-\sin h+I(x)+\xi(x, t),
$$

whose linear part is the already mentioned linear MBE equation [5,6], the biharmonic term describing diffusion of material along the surface.

A word is in order regarding the applicability of model (1),(2). A relevant context is of course epitaxial growth, by MBE or other vapor-phase techniques not operating in a vacuum environment [17]. Note that we are not introducing any term accounting for a step-edge barrier, $[1,4,17]$ which would give rise to surface tension and/or unstable growth; therefore, we are addressing situations in which time and length scales are small enough for surface tension to be negligible [18], and/ or the temperature range is high enough for step-edge barriers not to be relevant, both conditions yielding an effectively tensionless surface (alternatively, anisotropic diffusion and attachment can also balance step-edge barriers). An important case where such barriers are suppressed is, for instance, homoepitaxy onto low-index faces of silicon at room temperature [17]. This has been experimentally confirmed by Yang et al. in thermally evaporated Si films [8]; for sputter deposited Pt on glass, similar results have been obtained by Jeffries et al. [9]. Finally, we point out that Eq. (2) is a nonconserving one; if there is a conserved current of material along the surface, the periodic term representing lattice effects must be different $[4,19]$.

We now proceed with the main question about the equilibrium $\left(I_{i}=0\right)$ of our model: the existence or not of a roughening transition. As we know of no previous analytical or numerical results for Eqs. (2) and (3), we recall the renormalization group (RG) ideas applied to the sG equation $[10,20]$ : There is a roughening temperature $T_{R}$ above which the energy of a step on the surface is zero, because temperature "renormalizes" the sine term, effectively suppressing it at and above $T_{R}$; in turn, the lattice potential modifies the effective value of the surface tension coefficient [21]. In Fig. 1, we plot Langevin dynamics results for model (1) [with $h_{i}(t=$ $0)=0]$, namely, the surface structure factor $S(\boldsymbol{k}, t) \equiv$ $\langle\hat{h}(\boldsymbol{k}, t) \hat{h}(-\boldsymbol{k}, t)\rangle[\hat{h}(\underline{k, t})$ being the Fourier transform of $\left(h_{i}(t)-\overline{h(t)}\right)$, with $\overline{h(t)}$ the mean height; note that the surface roughness $\left.W^{2}(L, t)=\sum_{k} S(\boldsymbol{k}, t)\right]$. Above $T_{R} \approx$

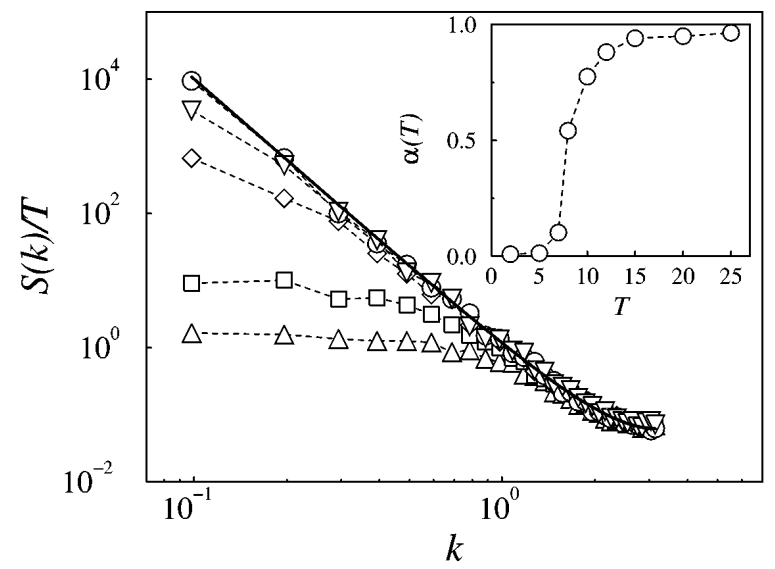

FIG. 1. $S(k, t)$ vs $k$ in the stationary regime for, bottom to top, $T=2,7,8,10$, and 20 (the last two ones overlap) and $L=64$. Dashed lines are guides to the eye. Solid line is the scaling of the discrete linear MBE equation, $S(k, t \rightarrow \infty) / T=1 /\left[16 \sin ^{4}(k / 2)\right]$. Inset: roughness exponent $\alpha$ (defined effectively from $W_{\text {sat }} \sim L^{\alpha}$ ) vs $T$. Error bars are of the symbol size or smaller.

$10, S(\boldsymbol{k}, t)$ scales in the stationary regime in the same fashion as for the linear MBE equation, $S(\boldsymbol{k}, t \rightarrow \infty) / T=$ $1 /\left[16 \sin ^{4}(k / 2)\right]$ [equivalently $W_{\text {sat }}(L) \equiv W(L, t \rightarrow \infty) \sim$ $L$ (see inset of Fig. 1)]. Below $T_{R}$, the surface is flat, i.e., $W_{\text {sat }}(L)$ does not depend on $L$, and $S(\boldsymbol{k}, t)$ does not exhibit any specific scaling. We thus identify $T_{R}$ as the temperature above which the sine term renormalizes to zero and Eq. (2) effectively behaves as the linear MBE equation. Furthermore, a variational calculation [22] (following that in [2] for the sG equation) yields $T_{R} \approx 12$, in very good qualitative agreement with Langevin dynamics. For the sake of comparison, for the sG model the roughening transition takes place at $T_{R}^{\mathrm{sG}}=8 \pi$ in our units $[10,16,20]$.

After finding $T_{R}$, we focus on the asymptotic scaling of the high temperature phase. An extension to our model [22] of the RG calculations for the sG model [20] suggests the presence of an effective, possibly small, surface tension term generated by the lattice potential [21]. If this is the case, the Laplacian term should dominate the scaling behavior [1] for $T>T_{R}$ after a crossover time $t_{\times}$(which increases with temperature) observable only for large enough system sizes due to saturation effects. The scaling for $t>t_{\times}$would then be that of the EdwardsWilkinson (EW) equation [23]. We stress that for $t<t_{\times}$ and $T>T_{R}$, our simulations yield the same dynamics as the linear MBE equation, i.e., $W(L, t) \sim t^{1 / 4}$. Although our present computing facilities do not allow us to unambiguously confirm the existence of asymptotic EW scaling (up to $L=512$ ), we obtained further evidence from the average velocity $v$ of an interface given by the transformation $h^{\prime}(x, t)=h(x, t)-\epsilon x^{2}$. In the presence of a finite Laplacian with coefficient $\nu, v$ behaves as $v \sim \nu \epsilon$ when $\epsilon \rightarrow 0$. We have obtained this behavior in our simulations, whose results support a small but 
distinctly nonzero value for $\nu$. Further work to settle this question is in progress [22].

Turning now to the nonequilibrium $\left(I_{i}=I \neq 0\right)$ case, Fig. 2 summarizes our results splitting the $(T, I)$ parameter space into three different regions. In region $A$, the lattice potential controls the dynamics, the surface is flat and shows zero average velocity like in the low $T$ phase at equilibrium. Increasing $I$ further for fixed $T$ within region $A$, a value of the driving is reached which is strong enough to pull the surface over the potential barriers, leading to a nonzero average velocity $v$ for the interface; hence the system is in region $B$. What is more interesting, increasing $T$ for fixed $I$ produces the same effect: This can be appreciated from Fig. 3, where the linear response mobility $\mu=v / I$ for $I=0.1$ exhibits a sharp transition from a pinned (region $A$ ) to a moving (region $B$ ) interface. The transition temperature decreases with increasing $I$; in fact, growth occurs for all $T$ when $I \geq 1$; that is, region $A$ terminates at $T=0, I=1$. This is seen also in Fig. 3 for $I=1.1$.

The difference between regions $B$ and $C$ relates not only to mobility but also to morphology. In region $C$, the mobility is maximum $(\mu \approx 1)$, and the interface displays the same scaling behavior as in the equilibrium high $T$ phase. On the contrary, region $B$ is characterized by a temperature dependent mobility: The interface moves nonlinearly, in a way which is reminiscent of the LBL growth experimentally observed in RHEED oscillations, particulary if one looks at the roughness dependence on time (upper panel of Fig. 4). To understand this behavior, let us recall that, for actual surfaces, there is a competition between surface diffusion, which tends to complete layers before new ones start to form, and the incoming flux, which supplies new material for island nucleation on top of incomplete layers. Consequently, three time scales arise: a surface diffusion time $t_{d}\left(t_{d} \sim \kappa^{-1}=1\right.$ in our units), a time associated with the flux rate $t_{I} \sim I^{-1}$, and a time associated with flux rate fluctuations $t_{T} \sim T^{-1 / 2}$. As $t_{d}$ is fixed, the relevant quantity for the dynamics

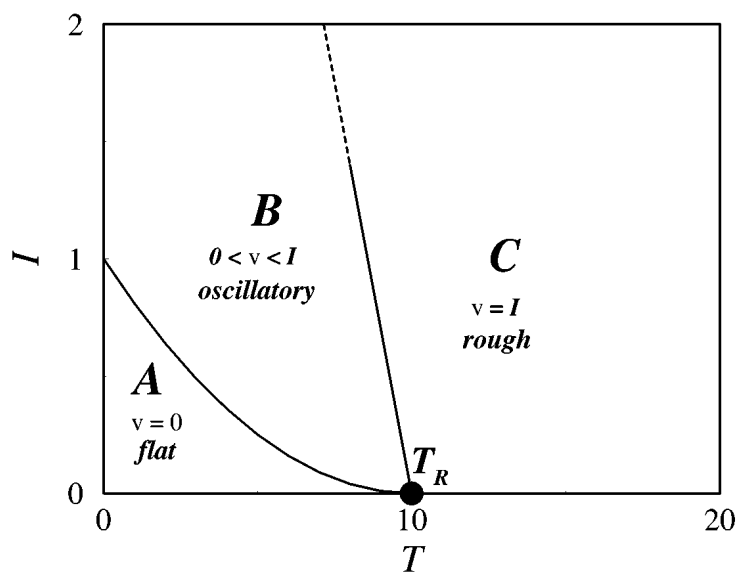

FIG. 2. Sketch of the "phase diagram" of our model. Lines are approximate and not intended as quantitatively correct, except for the ends of the line separating region $A$ from $B$. in region $B$ is the ratio $\gamma=t_{T} / t_{I}$ : Indeed, for large $\gamma$, the height fluctuations due to $T$ grow in a typical time scale $t_{T}$, much slower than the time needed by the incoming flux to pull the surface over a potential barrier, leading to LBL behavior. On the other hand, for small $\gamma$, temperature fluctuations dominate over the surface growth as a whole, yielding rough, multilayer growth. This is in perfect agreement with what we see in region $B$, where $\gamma$ decreases when going from region $A$ to region $C$. In addition, region $C$ shows no sign of oscillations, one more hint that the potential is effectively renormalized to zero by temperature.

For the purpose of comparison to experiments, we note that roughness oscillations in region $B$ of our model and RHEED oscillations can be related through the coverage $\theta_{n}$ of the $n$th layer, i.e., the number of occupied sites in that layer divided by the total number of available sites. The surface roughness reads $W^{2}=(2 \pi)^{2} \sum_{n}\left(\theta_{n}-\right.$ $\left.\theta_{n+1}\right)(n-\bar{h})^{2}$, where we have discretized the surface height values: $h_{n}=2 n \pi$. In perfect LBL growth, at any given time $\theta_{n}$ is different from 0 or 1 only for one value of $n$, and $W^{2}=(2 \pi)^{2}\left(\theta_{n}-\theta_{n}^{2}\right)$, oscillating between 0 and $\pi^{2}$ as $\theta_{n}$ goes from 0 to 1 . RHEED spectra, which are directly related to coverages [3], behave accordingly, oscillating with constant amplitude in time. On the contrary, when two or more layers are growing at the same time (rough growth), damping terms appear in $W^{2}$, and hence oscillations damp out (see Fig. 4), once again as RHEED oscillations do. In all cases, $\gamma$ determines the period of oscillations and the damping time. Region $B$ is thus the parameter range for which one would expect LBL growth (close to region $A$ ) crossing over to rough growth (as one approaches region $C$ ). In region $C$, the suppression of the lattice potential yields the very notion of layer meaningless.

In conclusion, we have proposed and studied a model for growth of crystalline tensionless surfaces [Eq. (1)] which shows an equilibrium roughening transition at

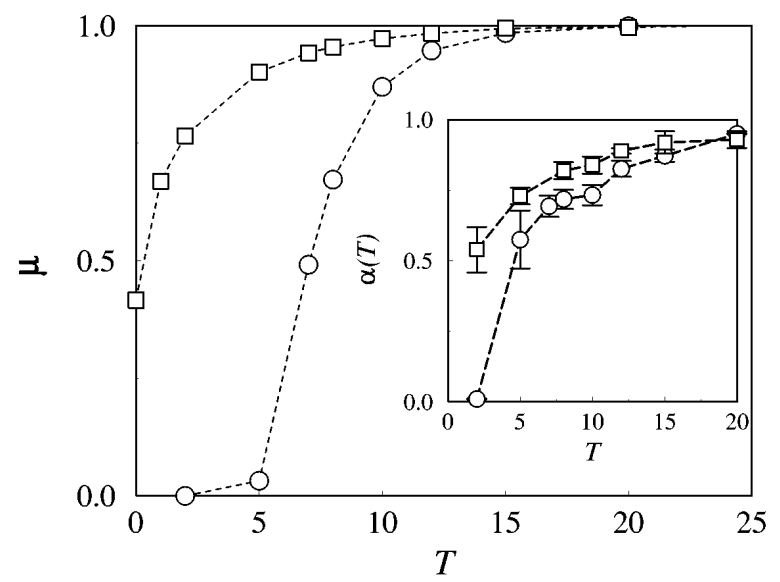

FIG. 3. Linear mobility $\mu \equiv v / I$ vs $T$ for $I=0.1$ (०) and 1.1 ( $\square$ ). Inset: roughness exponent $\alpha$ vs $T$ for the same values of $I$. Error bars are of the symbol size or smaller except where plotted. 


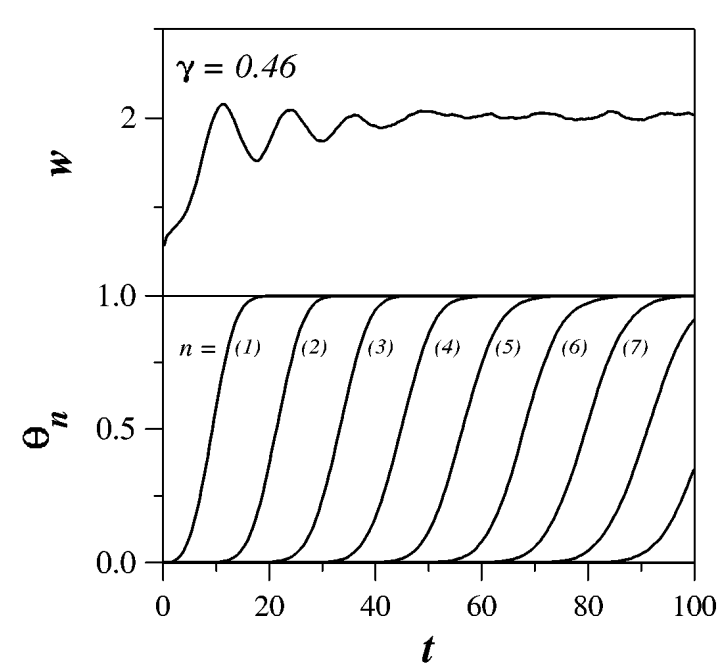

FIG. 4. $W$ (upper panel) and coverage of the first seven layers (lower panel) vs $t$ for $I=0.8$ and $T=3$ (region $B, \gamma=0.46$ ). Roughness oscillations damp out because several layers are growing simultaneously.

temperature $T_{R} \approx 10$ (dimensionless units), approximately half of that required by a system minimizing surface tension. The surface morphology at $T>T_{R}$ is much rougher, at least in the early dynamics, than in the case of surfaces with nonzero tension. Out of equilibrium, the phase diagram is composed of three regions where the surface is pinned, $A$, moves nonlinearly, $B$, or roughens kinetically, $C$. The nonlinear region $B$ is a crossover from LBL growth (close to the pinned phase $A$ ) to rough growth (close to the rough phase $C$ ). As we have discussed, this characterization is relevant to experiments, and real systems may exhibit measurable signatures of the three regimes. We note that, among those usually studied in the context of rough surfaces, no other continuum model without lattice effects has reproduced these oscillations [1]. Presently, reports of RHEED oscillations dependence on temperature are available and qualitatively agree with our results [24], although of course much more work is needed to assess the relevance of our model to actual growth processes. Finally, it is most important to clarify the asymptotic scaling of our model in the high temperature phase. We believe the asymptotic scaling for $T>T_{R}, I \geq 0$ is the same as for the $\mathrm{sG}$ model. If confirmed, this result implies that in realistic systems the linear MBE equation scaling cannot be asymptotic. However, the crossover time can be large, presumably observable for appropriate experimental realizations, such as, e.g., homoepitaxy by vapor techniques under properly chosen conditions.

We are indebted to L. A. N. Amaral, A. R. Bishop, J. A. Cuesta, E. Diez, and M. Kotrla for discussions. This work has been supported by CICyT (Spain) Grant No. MAT95-0325.

Note added. - After this Letter was accepted for publication, we became aware of work on a related discrete model on triangular, lattices introduced by D. R. Nelson (see references in [25]). We are currently studying the connection between those results and ours.

*Electronic address: eme@dulcinea.uc3m.es

†Electronic address: cuerno@dulcinea.uc3m.es

\#Electronic address: anxo@dulcinea.uc3m.es

[1] A.-L. Barabási and H. E. Stanley, Fractal Concepts in Surface Growth (Cambridge University, Cambridge, 1995).

[2] S.A. Safran, Statistical Thermodynamics of Surfaces, Interfaces, and Membranes (Addison Wesley, Reading, MA, 1994).

[3] Kinetics of Ordering and Growth at Surfaces, edited by M. G. Lagally, NATO ASI Ser. B, Vol. 239 (Plenum, New York, 1990), and references therein.

[4] J. Krug, Adv. Phys. 46, 139 (1997).

[5] J. Villain, J. Phys. I (France) 1, 19 (1991).

[6] Z.-W. Lai and S. Das Sarma, Phys. Rev. Lett. 66, 2348 (1991).

[7] C. Herring, J. Appl. Phys. 21, 301 (1950); W. W. Mullins, J. Appl. Phys. 28, 333 (1957).

[8] H.-N. Yang, Y.-P. Zhao, G.-C. Wang, and T.-M. Lu, Phys. Rev. Lett. 76, 3774 (1996).

[9] J. H. Jeffries, J.-K. Zuo, and M. M. Craig, Phys. Rev. Lett. 76, 4931 (1996).

[10] S. T. Chui and J. D. Weeks, Phys. Rev. B 14, 4978 (1976); Phys. Rev. Lett. 40, 733 (1978).

[11] J. D. Weeks and G. H. Gilmer, Adv. Chem. Phys. 40, 157 (1979).

[12] A. C. Levi and M. Kotrla, J. Phys. Condens. Matter 9, 299 (1997).

[13] The Hamiltonian of the discrete Gaussian model is $\mathcal{H}_{d G}=(\nu / 2) \sum_{\mathrm{nn}}\left(h_{j} \quad h_{i}\right)^{2}$.

[14] The discrete $s G$ equation is Eq. (2) with a Laplacian instead of a bi-Laplacian (see [10]). The corresponding continuum $\mathrm{sG}$ equation in the overdamped limit reads $\frac{\partial h}{\partial t}=\nu \nabla^{2} h \quad \sin h+I(x)+\xi(x, t)$. Here $\nu$ is a surface tension coefficient related to surface stiffness.

[15] G. Grinstein and D.-H. Lee, Phys. Rev. Lett. 66, 177 (1991).

[16] F. Falo, A. R. Bishop, P. S. Lomdahl, and B. Horovitz, Phys. Rev. B 43, 8081 (1991); A. Sánchez, D. Cai, N. Grønbech-Jensen, A. R. Bishop, and Z. J. Wang, Phys. Rev. B 51, 14664 (1995).

[17] A. Zangwill, J. Cryst. Growth 163, 8 (1996).

[18] D. D. Vvedensky, A. Zangwill, C.N. Luse, and M.R. Wilby, Phys. Rev. E 48, 852 (1993).

[19] L.-H. Tang and T. Natterman, Phys. Rev. Lett. 66, 2899 (1991).

[20] P. Nozières and F. Gallet, J. Phys. (Paris) 48, 353 (1987).

[21] That is, a Laplacian term like that in [14] would be generated in the effective equation of motion for a coarse grained system.

[22] E. Moro, R. Cuerno, and A. Sánchez (to be published).

[23] S. F. Edwards and D. R. Wilkinson, Proc. R. Soc. London A 381, 17 (1982).

[24] A. S. Arrott, B. Heinrich, and S. T. Purcell, in Kinetics of Ordering and Growth at Surfaces (Ref. [3]).

[25] K. J. Strandburg, Rev. Mod. Phys. 60, 161 (1988). 